\title{
Abdominal Pain After Subtotal Gastrectomy: A First Report of Accessory Pancreatic Fistula
}

This article was published in the following Dove Press journal:

Journal of Pain Research

\author{
Jia-Yu Zhang (D) \\ Jia Huang ${ }^{2}$ \\ Zhi-Ying Yang ${ }^{1,2}$ \\ 'Graduate School of Peking Union \\ Medical College, Beijing 100029, People's \\ Republic of China; ${ }^{2}$ Department of \\ General Surgery, China-Japan Friendship \\ Hospital, Beijing I00029, People's \\ Republic of China
}

Background: The accessory pancreatic duct (APD) is the main drainage duct of the dorsal pancreatic bud in the embryo and varies greatly during development. An APD fistula is a rare and easily neglected complication. In this case report, the first symptom of the patient was postoperative abdominal pain and fever. He was eventually diagnosed with accessory pancreatic fistula combined with duodenal fistula. Such a case has not been reported in the literature.

Case Summary: A 66-year-old man was emergently hospitalized for abdominal pain. His preliminary diagnosis was perforation of the digestive tract. He developed fever and abdominal pain after emergency subtotal gastrectomy, followed by changes in the colour of the abdominal drainage fluid. An APD fistula and duodenal stump fistula were confirmed by drainage fluid amylase analysis, contrast fistulography and percutaneous transhepatic cholangial drainage (PTCD). After PTCD, nutritional management and drug treatment, the patient recovered well.

Outcome: We found and successfully cured a case of accessory pancreatic duct fistula combined with duodenal stump fistula.

Keywords: gastrointestinal perforation, abdominal pain, accessory pancreatic duct fistula, duodenal stump fistula, case report

\section{Introduction}

The accessory pancreatic duct (APD) is the main drainage duct of the dorsal pancreatic bud in the embryo and varies greatly during development. ${ }^{1}$ It is generally believed that the APD is located in the upper part of the head of the pancreas, is generally relatively short, and travels in the anterior and upper part of the pancreatic duct, mainly draining the pancreatic juice from the upper and anterior part of the head of the pancreas. Duodenal stump fistula (DSF) is an uncommon but extremely dangerous complication after gastric surgery and is one of the most challenging and life-threatening postoperative events that the surgeon must deal with. The DSF incidence is about $3 \%$, overall mortality ranges from $7 \%$ to $67 \%$. $^{2}$ Infections (pneumonia, abdominal abscess, surgical site infection) and subsequent sepsis are the most common mortality causes. APD fistula combined with DSF is a rare and potentially fatal diagnosis, and no such case has been reported in the literature.

\section{Ethics}

The patient provided a written informed consent allowing the publication of the case details and accompanying images; the privacy of the patient was maintained
Correspondence: Zhi-Ying Yang Department of General Surgery, ChinaJapan Friendship Hospital, 2 Cherry Blossom East Street, Beijing 100029, People's Republic of China Email yangzhy@aliyun.com 
with confidentiality. Due to the retrospective nature of the case, institutional approval was not required.

\section{Case Report}

The patient was a 66-year-old male. He was hospitalized for "paroxysmal abdominal pain and distention accompanied by cessation of flatus and defecation for 2 days". Epigastric paroxysmal pain had aggravated and spread to the whole abdomen since the onset of illness. The patient was nauseous and vomited several times. He received conservative antiinflammatory and analgesic treatments in the local hospital for 2 days without remission. He had a history of hypertension and ankylosing spondylitis. Physical examination revealed epigastric tenderness with rebound pain and muscle tension; tympany in the abdomen; The disappearance of normal liver dullness; and weakened intestinal sounds. Laboratory examination showed the following: white blood cells (WBCs), 16.84*10\%/L; procalcitonin (PCT), $10.02 \mathrm{ng} /$ $\mathrm{mL}$, creatinine kinase-MB fraction (CK-MB), $8.50 \mathrm{ng} / \mathrm{mL}$; and N-terminal pro-brain natriuretic peptide (NT-proBNP), $1263 \mathrm{pg} / \mathrm{mL}$. Abdominal CT revealed a large amount of free gas in the abdominal cavity, probable gastrointestinal perforation; a right renal cyst; and ankylosing spondylitis. The patient's admission diagnosis was as follows: (1) gastrointestinal perforation and (2) intestinal obstruction. The patient underwent laparotomy immediately after admission, and a large perforation of the anterior wall of the duodenal bulb was found during the operation. Subtotal distal gastrectomy, $\mathrm{R}-\mathrm{Y}$ gastrojejunostomy, and abdominal lavage and drainage

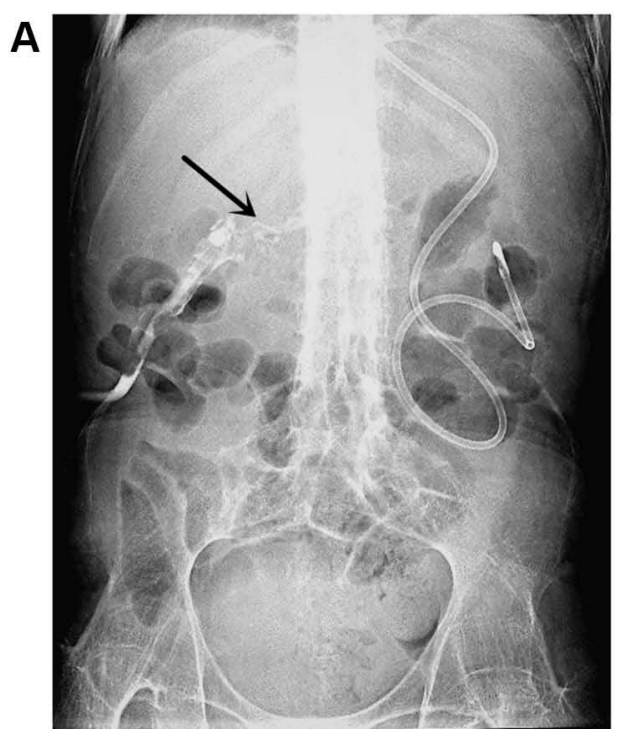

were performed. Drainage tubes were placed in the posterior liver, splenic fossa, pelvic cavity and duodenal stump after the operation. Among them, the indwelling drainage tubes in the posterior liver, splenic fossa and pelvic cavity drained smoothly, and the drainage volume gradually decreased. After gradual withdrawal of the drainage tubes, the drainage tubes were individually removed. The drainage fluid from the duodenal stump drainage tube was initially light red and decreased to $100 \mathrm{~mL}$ on the 8th day after the operation. The patient was then given permission to start consuming liquid food after exhaust. After two days of consuming a liquid diet, the patient developed abdominal pain and fever. The drainage fluid increased to $250 \mathrm{~mL}$, and the colour of the drainage fluid became clear and transparent. Amylase in the drainage fluid was $14420 \mathrm{IU} / \mathrm{L}$ during an emergency examination. The patient was then fasted and parenteral nutrition was administered. At the same time, somatostatin and antibiotics were given as adjuvant therapies. The drainage fluid turned light green on the 11th day and dark green on the 12th day after the operation, and the drainage volume increased continuously. So we highly suspected DSF. A contrast agent was injected through the duodenal stump drainage tube 13 days after the operation. Abdominal plain films showed that the contrast agent entered the abdominal cavity, and an APD was first displayed, then the main pancreatic duct and common bile duct showed up (Figure 1). A small amount of contrast agent can enter the duodenum. This suggested that the APD was connected to the abdominal cavity and that an APD fistula may exist. Percutaneous

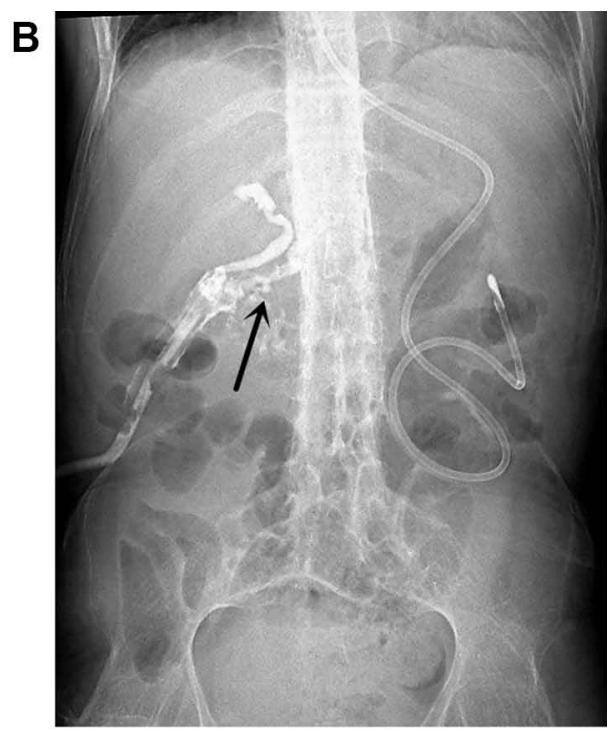

Figure I Abdominal plain films after injecting of a contrast agent.

Notes: APD was displayed, as marked by the black arrow in (A). The main pancreatic duct and common bile duct showed up in (B). 
transhepatic cholangial drainage (PTCD) and digital subtraction angiography (DSA) were performed at the same time (Figure 2). The guide wire entered the duodenal stump drainage tube through the biliary tract during the operation. After repeated manipulation, the guide wire avoided the duodenal stump area and entered the duodenum. Then, $8.5 \mathrm{~F}$ internal and external drainage tubes were placed according to the guide wire, and the contrast agent indicated good drainage. After PTCD, the patient returned to the ward in good condition. Enteral nutrition was increased while the amount of parenteral nutrition was decreased. Additionally, we washed the drainage tube which besides the duodenal stump with a slow drip of $1000 \mathrm{~mL}$ of normal saline for two days and paid close attention to changes of drainage fluid. Subsequently, the average daily drainage volume of the PTCD tube was approximately $800 \mathrm{~mL}$. The drainage volume of the duodenal stump was significantly lower than that before PTCD, averaging about $50 \mathrm{~mL}$ per day. The drainage volume of the duodenal stump decreased to 0 on the 7th day after PTCD. So we intermittently clamped the PTCD tube for two days and then continuously clamped the PTCD tube for one week, during which we paid close attention to the drainage and general conditions of the patient. The

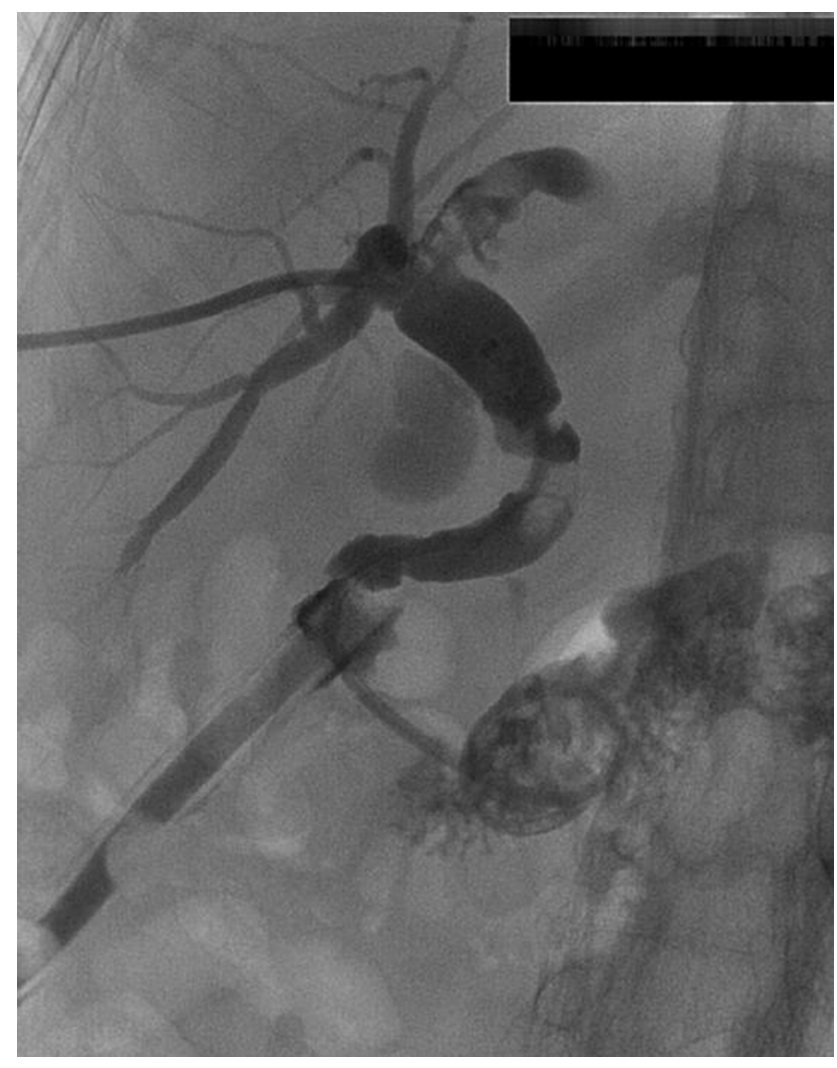

Figure 2 PTCD and DSA. patient's food intake and body temperature were normal, and the drainage volume from the drainage tube near the duodenal stump did not increase again, so we gradually retreated and eventually removed the drainage tube at the duodenal stump. The PTCD tube was removed after two weeks of observation. We followed the patient at 1, 3, and 6 months after discharge, and the patient recovered well.

\section{Discussion}

Gastrointestinal perforation is a common disease of the digestive tract system in the clinic. It is often secondary to gastrointestinal ulcer, severe inflammatory bowel disease, diverticulitis, gastrointestinal cancer, intestinal obstruction trauma and closed loop intestinal obstruction. ${ }^{3}$ Gastrointestinal perforation often has abdominal distension and different degrees of abdominal pain in the early stage. If not treated in time, it may eventually lead to severe sepsis, which may lead to multiple organ failure and even death. However, from the point of view of clinical practice, the medical history, signs, and symptoms of digestive tract perforation are often atypical, which causes difficulties in clinical diagnosis and delays the opportunity for treatment. ${ }^{4}$ The patient's condition was aggravated because the local hospital failed to accurately diagnose and identify the indication for operation in the early stage of the disease, which increased the difficulty of the operation and the risk of complications. After completing the necessary examinations and identifying the surgical indications, the patient underwent emergency subtotal gastrectomy, gastro-jejunal R-Y anastomosis, and abdominal lavage and drainage. The patient's drainage fluid amylase was $14420 \mathrm{IU} / \mathrm{L}$ on the 10th day after surgery. According to the definition of the International Study Group for Pancreatic Fistulas (ISGPF), the patient has reached the diagnostic criteria for postoperative pancreatic fistula. ${ }^{5}$ Therefore, contrast fistulography was performed after the operation. The contrast agent first entered the APD, suggesting an APD fistula but not excluding concurrent DSF, which is very rare in the clinic and has not been previously reported. For the diagnosis of APD fistula, endoscopic retrograde cholangiopancreatography (ERCP) can provide direct evidence; the sensitivity and specificity for the diagnosis of pancreatic duct rupture are both $100 \%{ }^{6}$ However, it was difficult to perform ERCP in this patient because he had undergone subtotal gastrectomy and R-Y anastomosis. In addition, ERCP requires sedation and there is a risk of duodenal perforation and pancreatitis. Abdominal CT has a low sensitivity for the diagnosis of APD fistula and is 
mainly used to exclude other causes of abdominal pain. It can also show free and encapsulated effusion in the abdominal cavity. Magnetic resonance cholangiopancreatography (MRCP) enables the noninvasive detection and exclusion of pancreatic duct injury and pancreas-specific complications and provides information that can be used to guide management decisions, but MRCP cannot be used for treatment. ${ }^{7}$ Usually, we use only contrast fistulography to determine the specific condition and location of the fistula when ERCP/MRCP cannot clearly identify the APD fistula. However, for patients with a postoperative pancreatic fistula requiring drainage, contrast fistulography is superior to ERCP and MRCP. Fistulography can also show the associated fluid accumulation and can guide the repositioning of the drainage tube to achieve maximum drainage. Due to a suspicious APD fistula combined with DSF and increasing abdominal drainage, we performed contrast fistulography during PTCD and found the presence of an APD fistula. As seen in Figure 1A and B, the APD and the main pancreatic duct indicated by the arrows had different directions of travel, which also helped us to diagnose the APD fistula. Unfortunately, for a variety of reasons, we did not perform MRCP after PTCD to further confirm the diagnosis of APD fistula. However, through this case report, we further recognized the importance of MRCP in the diagnosis of pancreatic-related diseases. Therefore, for cases of suspected APD fistula after such gastric surgery, we recommend combining MRCP and contrast fistulography to confirm the diagnosis.

The APD is the main drainage duct of the dorsal pancreatic bud in the embryo and varies greatly during development. ${ }^{1}$ It is generally believed that the APD is located in the upper part of the head of the pancreas, is relatively short, and travels in the anterior and upper part of the pancreatic duct, mainly draining the pancreatic juice from the upper and anterior part of the head of the pancreas. The left side of the APD often converges with the main pancreatic duct, and the right side mostly opens directly to the minor duodenal papilla. Possible risk factors of APD fistula include gender, diabetes, body mass index (BMI), blood glucose level, age, time of onset before the operation, degree of tissue oedema around the perforation, the diameter of the pancreatic duct, pancreatic texture, and operation time. ${ }^{8}$ In this case, due to the patient's age, obvious oedema of the surrounding tissues during operation and severe intraperitoneal contamination, the minor duodenal papilla may have been torn when the duodenum was separated. The pancreatic juice leaked from the fistula after operation, corroded the surrounding tissue and formed a sinus connecting to the abdominal drainage tube. The authors believe that an APD fistula should be treated similarly to a relatively common pancreatic fistula because it has not been reported previously. Fasting with nutritional support, adequate drainage, and skin protection are currently the basic aspects of treatment for pancreatic fistula. ${ }^{9}$ Because the previously transparent abdominal drainage fluid turned dark green on the 12th day after the operation and was mixed with a small amount of intestinal fluid, we suspected a concomitant DSF. In addition, the abdominal drainage tube in this patient happened to be parallel to the common bile duct. When PTCD was performed on the 22nd day after the operation, the guide wire accidentally entered the abdominal drainage tube through the major duodenal papilla, suggesting the possibility of DSF. DSF is an uncommon but extremely dangerous complication after gastric surgery and represents one of the most challenging and life-threatening postoperative events that the surgeons must deal with. The DSF incidence is about $3 \%$, and overall mortality ranges from $7 \%$ to $67 \%{ }^{2}$ Infections (pneumonia, abdominal abscess, and surgical site infections) and subsequent sepsis are the most common mortality causes observed. Possible causes of DSF include local haematoma, inflammation, poor closure of the duodenal stump during operation, incorrect drainage position, and duodenal dilatation after the operation. Aurello et $\mathrm{al}^{10}$ showed in a large study that conservative treatment should be the preferred treatment for DSF. Surgery should be considered only when other methods fail or DSF results in serious complications, such as severe bleeding or sepsis. According to the literature, percutaneous biliary drainage can be used to reduce leakage in patients with severe systemic conditions, persistent DSF or high daily fluid leakage. ${ }^{10}$ After internal and external PTCD, the abdominal drainage tube was washed with a slow drip of normal saline for several days. The leakage was significantly reduced, and the colour of the drainage fluid returned to clear. After gradual withdrawal, the drainage tube was removed from the duodenal stump. The nutritional status of patients is also an important factor affecting the healing of DSF and APD fistula. Different nutritional methods may affect the healing of DSFs. At present, both enteral and parenteral nutrition for the treatment of pancreatic fistula have been reported. In contrast with parenteral nutrition, enteral feeding generally not only avoids pancreatic stimulation but also stimulates the release of specific gut peptides, forming a negative feedback control system and thus inhibiting pancreatic secretion. In conclusion, enteral nutrition appears to be an effective, safe, and cost-effective therapeutic option in 
patients with postoperative pancreatic fistula. ${ }^{9}$ Besides, studies have shown that perioperative treatment with pasireotide decreases the rate of clinically significant postoperative pancreatic fistula, ${ }^{11}$ but it is still controversial. Subtotal gastrectomy for perforation of gastrointestinal ulcers is the most commonly used treatment method in China, as it can address perforations and ulcers at the same time. According to the literature, $90 \%$ of the short-term results after subtotal gastrectomy were satisfactory. However, the occurrence of various complications has attracted increasing attention from surgeons, and DSF is one of the serious complications. APD fistula and DSF occurred at the same time in this patient; this phenomenon has not been reported in domestic or foreign literature. In such cases, we believe the most important factor is to reduce the difficulty of the operation and the probability of complications by the early definitive diagnosis and the timely identification of surgical indications. At the same time, individualized surgical techniques according to the patient's specific condition should also be considered. According to research, the selection of the appropriate staple height and reinforcement of the staple line could play a major role in optimizing the results of gastrectomy. ${ }^{12}$ For unexplained abdominal pain and fever after gastrointestinal surgery, we should consider the possibility of APD fistula. Once APD fistula and DSF occur, internal and external PTCD combined with personalized nutritional support is an effective treatment strategy.

\section{Author Contributions}

All authors contributed to data analysis, drafting or revising the article, gave final approval of the version to be published, and agree to be accountable for all aspects of the work.

\section{Funding}

This work was financially supported by the Fund of ChinaJapan Friendship Hospital (No. 2017-RC-2).

\section{Disclosure}

The authors report no conflicts of interest in this work.

\section{References}

1. Kamisawa T, Takuma K, Tabata T, Egawa N. Clinical implications of accessory pancreatic duct. World J Gastroentero. 2010;16:4499. doi:10.3748/wjg.v16.i36.4499

2. Orsenigo E, Bissolati M, Socci C, et al. Duodenal stump fistula after gastric surgery for malignancies: a retrospective analysis of risk factors in a single centre experience. Gastric Cancer. 2014;17:733-744. doi:10.1007/s10120-013-0327-x

3. Winek TG, Mosely HS, Grout G, Luallin D. Pneumoperitoneum and its association with ruptured abdominal viscus. Arch Surg. 1988;123:709-712. doi:10.1001/archsurg.1988.01400300051008

4. Nicolodi GC, Trippia CR, Caboclo MF, et al. Intestinal perforation by an ingested foreign body. Radiol Bras. 2016;49:295-299. doi:10.1590/0100-3984.2015.0127

5. Bassi C, Dervenis C, Butturini G, et al. Postoperative pancreatic fistula: an international study group (ISGPF) definition. Surgery. 2005;138:8-13. doi:10.1016/j.surg.2005.05.001

6. Rogers SJ, Cello JP, Schecter WP. Endoscopic retrograde cholangiopancreatography in patients with pancreatic trauma. $J$ Trauma. 2010;68:538-544. doi:10.1097/TA.0b013e3181b5db7a

7. Fulcher AS, Turner MA, Yelon JA, et al. Magnetic resonance cholangiopancreatography (MRCP) in the assessment of pancreatic duct trauma and its sequelae: preliminary findings. $J$ Trauma. 2000;48:1001-1007. doi:10.1097/00005373-200006000-00002

8. Hu B, Wan T, Zhang W, Dong J. Risk factors for postoperative pancreatic fistula: analysis of 539 successive cases of pancreaticoduodenectomy. World J Gastroentero. 2016;22:7797. doi:10.3748/wjg.v22.i34.7797

9. Klek S, Sierzega M, Turczynowski L, Szybinski P, Szczepanek K, Kulig J. Enteral and parenteral nutrition in the conservative treatment of pancreatic fistula: a randomized clinical trial. Gastroenterology. 2011;141:157-163. doi:10.1053/j.gastro.2011.03.040

10. Aurello P, Sirimarco D, Magistri P, et al. Management of duodenal stump fistula after gastrectomy for gastric cancer: systematic review. World J Gastroentero. 2015;21:7571. doi:10.3748/wjg.v21.i24.7571

11. Allen PJ, Gönen M, Brennan MF, et al. Pasireotide for postoperative pancreatic fistula. $N$ Engl J Med. 2014;371:875-876. doi:10.1056/ NEJMc 1407470

12. Debs T, Petrucciani N, Kassir R, et al. Complications after laparoscopic sleeve gastrectomy: can we approach a $0 \%$ rate using the largest staple height with reinforcement all along the staple line? Short-term results and technical considerations. Surg Obes Relat Dis. 2018;14(12):1804-1810. doi:10.1016/j.soard. 2018.08.028.
Journal of Pain Research

\section{Publish your work in this journal}

The Journal of Pain Research is an international, peer reviewed, open access, online journal that welcomes laboratory and clinical findings in the fields of pain research and the prevention and management of pain. Original research, reviews, symposium reports, hypothesis formation and commentaries are all considered for publication. The manuscript management system is completely online and includes a very quick and fair peer-review system, which is all easy to use. Visit http:// www.dovepress.com/testimonials.php to read real quotes from published authors. 